\title{
Visualization of Gubernacular Tract of Transmigrated Canine on CBCT
}

\author{
Astha Chaudhry ${ }^{1}$, Geetika Sobti² \\ ${ }^{1}$ Department of Oral Medicine and Radiology, Shree Guru Gobind Singh Tricentenary University, Gurugram, Haryana, \\ India. ${ }^{2}$ Department of Oral Medicine and Radiology, Government Dental College and Hospital, Jaipur, Rajasthan, India.
}

\section{INTRODUCTION}

Eruption of tooth is a physiologic process wherein the developing tooth migrates from its developmental position to the functional position in the alveolar process. Multiple theories have been postulated but the most widely accepted one is the asymmetric bone remodelling around the tooth brought about by the dental follicle. ${ }^{1,2,3}$

According to this, during the intra-osseous eruptive phase of the tooth eruption, an eruptive path is formed which guides the tooth to its eruptive position. This eruptive path is the Gubernacular tract (GT) or Gubernaculum dentis or Gubernacular canal which houses the Gubernacular cord. Gubernacular cord is composed of fibrous connective tissue containing peripheral nerves, blood and lymphatic vessels as well as epithelial cells and cell rests of Dental lamina. ${ }^{4}$ This cord also houses multiple chemical mediators including $\mathrm{EGF}^{4}$ (Epithelial Growth Factor) which have the capacity to stimulate osteoclast formation thereby causing bone resorption and hence widening of the canal as the tooth migrates closer to the alveolar process.

The first reference of Gubernacular cord and canal was done in 1778 by John Hunter ${ }^{5}$ when a connection was observed between the developing tooth and the gingiva and it was supported in microscopic studies by Louis-Charles Malassez ${ }^{6}$ in 1887 when epithelial cell rests of dental lamina along with longitudinal fibres were observed in the canal connecting the developing tooth with the overlying gingiva. The demonstration of Gubernacular tract or canal on radiographs is uncommon and has not been well documented in literature. One of the reasons for this is that these canals are very thin with a diameter of only about 1 to $3 \mathrm{~mm}$ and these lie in the spongy bone unrelated to the cortices of the maxilla and mandible. Hence, their representation on conventional 2-D imaging modalities was difficult and did not receive much attention. ${ }^{7}$ It is only with the advent of advanced imaging modalities like CBCT which allow precise evaluation of minute structures that this structure has started receiving attention by the radiologists. However, there are very few reports in the literature that discusses the detection rate of GT and its association with impacted teeth, odontogenic cysts and tumours.

Transmigration is the term coined by Ando et al 8 in 1964 which refers to the intraosseous migration of unerupted teeth across the midline. It is a rare phenomenon and is observed affecting the mandibular canines frequently as opposed to other teeth.

\author{
Corresponding Author: \\ Astha Chaudhry, \\ Flat No. 102, Tower G, \\ Corona Optus, Sec-37C \\ Gurugram, Haryana, India. \\ E-mail: dr.asthac@gmail.com
}

DOI: $10.14260 / j e m d s / 2019 / 785$

Financial or Other Competing Interests: None.

How to Cite This Article:

Chaudhry A, Sobti G. Visualization of gubernacular tract of transmigrated canine on CBCT. J. Evolution Med. Dent. Sci. 2019;8(48):3637-3640,

10.14260/jemds/2019/785

DOI:

Submission 31-08-2019,

Peer Review 11-11-2019,

Acceptance 18-11-2019,

Published 02-12-2019. 
The demonstration of GT in impacted teeth is rare and its demonstration in teeth which have migrated intra-osseously is even rare. There has been only one report in literature in 2019 which discusses the GT in a transmigrated canine ${ }^{9}$ but did not mention the alterations or characteristics of the GT. The present case shall be the second report of demonstration of GT in a transmigrated mandibular canine on CBCT.

The length of GT has not been discussed much in the literature. In one unpublished dissertation by Arajuo et al, the greatest length of GT as shown by teeth with altered eruption or impaction was only $11 \mathrm{~mm}$ so the present case shall be the first report to show the intact GT of length of $22.3 \mathrm{~mm}$.

The Gubernacular tract (GT) or Gubernaculum Dentis (GD) is an eruptive pathway from dental follicle to the gingiva of the permanent teeth. It is a canal containing the Gubernacular cord which is a fibrous band that connects the follicular sac of the permanent tooth with the overlying gingiva.

The visualization of GT on conventional radiographs is rare. However, with the advanced imaging modalities like CBCT, its visualization has improved. The demonstration of GT in association with impacted teeth or in teeth with altered eruption process is less and its association with transmigrated canine is very rare with only one case been reported so far. This present case shall be the second reported case of GT or altered eruption tract of transmigrated mandibular canine on CBCT but shall be the first case report showing an altered but intact GT of considerable length of $22.3 \mathrm{~mm}$.

\section{PRESENTATION OF CASE}

A 25-year-old female patient reported to the Dental institute for the CBCT evaluation of multilocular radiolucent lesion in the right mandible. The medical history was unremarkable. CBCT was performed using Carestream CS9300 imaging system consisting of an X-ray tube, a source collimator and an amorphous-silicon flat-panel detector (CS 3D Imaging v3.5.7; Carestream Health, Inc New York, USA) with the field of view (FOV) of $17 x 13 \mathrm{~cm}$. Image volume was reconstructed with isotropic isometric $300 \times 300 \times 300 \mu \mathrm{m}$ voxels. CBCT scans were analysed in a darkened quiet room on the Dell Precision workstation which comprised of dual monitors (Dell 24-inch nonglossy monitor (1920 x1200 resolution) using CS 3D software (v3.5.7) and appropriate slices including axial, panoramic and sagittal images through multiplanar reconstruction methods were obtained. Apart from the odontogenic keratocyst in the right body and angle region of the mandible, another interesting observation was Transmigrated 33 (Type 2) horizontally impacted below the apices of incisors and in approximation with the root apex of 43. A well-defined linear hypodense canalicular structure with parallel corticated walls was observed running from the lateral aspect of follicle of 33 till the alveolar ridge with respect to 34 with an approximate length of about $22.3 \mathrm{~mm}$ in the reformatted panoramic images (Fig. 1). On the sagittal images, it was visualized as a round hypodense structure of $1.5 \mathrm{~mm}$ width with corticated borders located on the labial aspect of mandibular incisors approximating the apices and above the impacted transmigrated mandibular canine (Fig. 2). The transmigrated canine was also seen causing impingement with near perforation of the labial cortical plate (Fig. 3). The visualizations of the tract were better in slice thickness of
$300 \mu \mathrm{m}$. The differential diagnosis included bony trabeculae, accessory mandibular canal, nutrient canals and sinus tract. Since, the dimensions were larger than the bone trabeculae and its association with the asymptomatic impacted teeth and the lack of association with mandibular canal, all other possibilities were excluded and the radiographic diagnosis of Gubernacular tract of impacted transmigrated canine in relation to 33 was made. The patient underwent the surgical treatment of the odontogenic keratocyst in the right mandible. The lack of symptoms associated with the tract and the unwillingness of the patient for the extraction of the transmigrated tooth did not allow us to study the histological aspects of the tract.

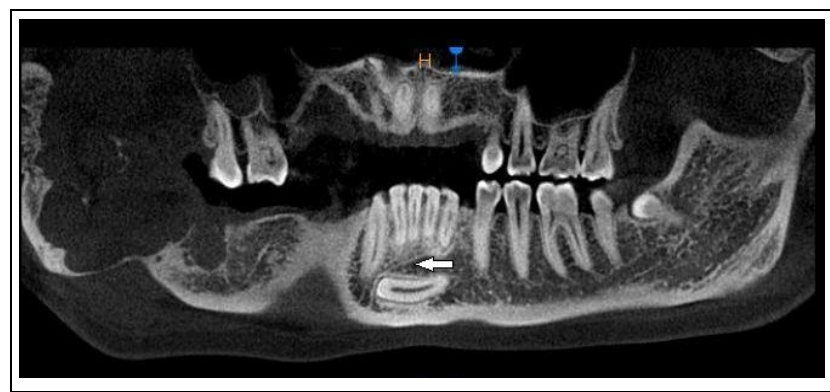

Figure 1. The Panoramic Section of CBCT Image Showing the Canalicular GT in Association with Transmigrated 33 of Length of $22.3 \mathrm{~mm}$.

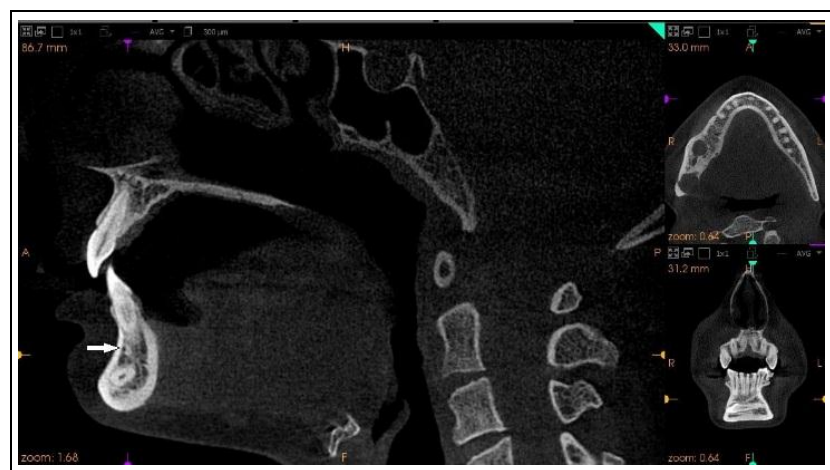

Figure 2. Sagittal Section Showing GT as Round Hypodense Structure of Width 1.5mm Located Coronal to the Horizontally Impacted 33 and Labial to the Apices of the Incisors with Corticated Borders

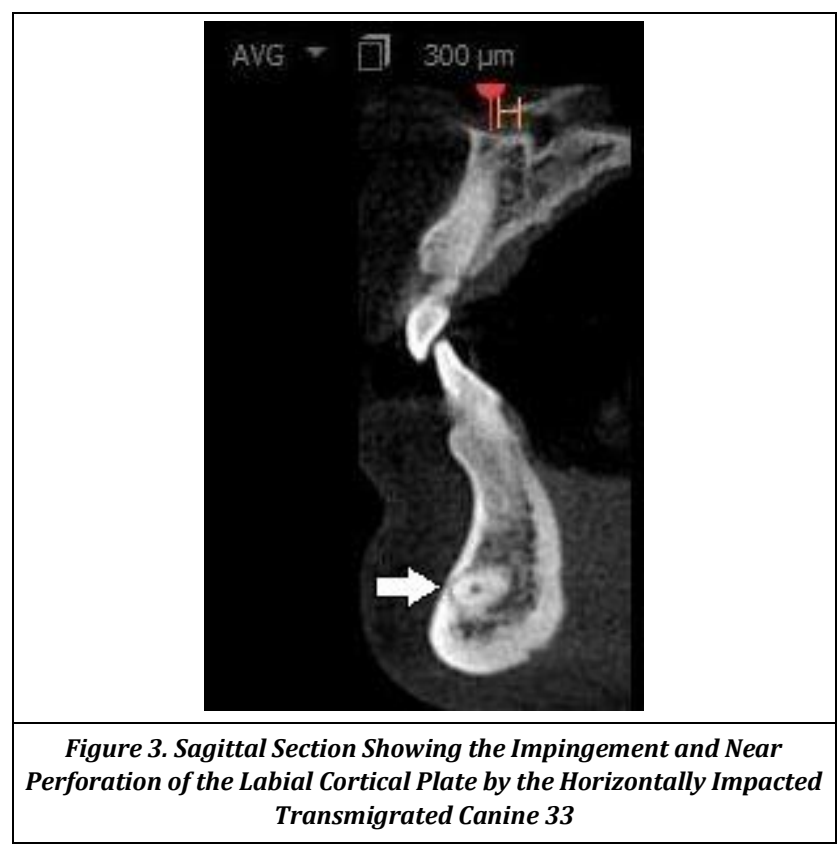




\section{DISCUSSION}

Gubernacular tract or Gubernacular canal is a normal anatomic structure housing the Gubernacular cord which plays an important role in guiding the tooth to its eruption position. According to Hodson, this canal has been described in the earlier literature to be associated only with succedaneous teeth. ${ }^{10}$ However, the permanent molar teeth without a deciduous predecessor also have their follicles connected to the mucosa by "Molar gubernacular cords".11 The recent studies by Oda et al,12,13 Nishida et $\mathrm{al}^{7}$ and Koc et al ${ }^{14}$ have also refuted the Hodson's concept with the demonstration of GT in association with impacted teeth, supernumerary mesiodens and odontogenic cysts and tumours.

With the advent of advanced imaging modalities like CBCT, the detection rates of GT (In the unpublished dissertation by Arajuo et al) have been shown to be $94.1 \%$ in teeth with normal eruption and about $87.1 \%$ in impacted teeth.

Koc et $\mathrm{al}^{14}$ have also mentioned that the detection rates of GT are significantly lower in cases with eruption disturbances. Also according to Nishida et $\mathrm{al}^{7}$ altered eruption processes in teeth are associated with the alterations in the GT which can be seen as a shift or deformation or displacement of GT.

Transmigration is an altered eruptive process of a tooth during which the tooth migrates intra-osseously with varied presentations. This is commonly encountered with mandibular canines and to simplify the diversity, a classification of transmigrated mandibular canines has been proposed by Mupparapu et al.15 According to this classification, the case reported was of type 2 category showing horizontal impaction to a considerable extent in the opposite direction crossing the midline. There was no obstruction or deformation of GT in the present case but it was altered in its inclination and showed its connection with the migrating tooth. The altered GT was seen running superoinferiorly from the alveolar ridge irt 34 and mediolaterally till 42 towards the labial aspect and also maintaining its connection with the supero-lateral aspect of follicle of impacted 33 with a total length of tract being $22.3 \mathrm{~mm}$. The determination of intact GT tract of such length is an exceptional finding and barely reported in literature. Whether this altered GT was the result of migration of tooth or this alteration in GT caused the transmigration is the research question that needs to be studied in longitudinal studies.

Though the authors claim that this is the second reported case of GT in transmigrated canine, but on critically reviewing the literature about CBCT study of transmigrated canines, some interesting observations were made. In the report by Gruszka et al,16 similar appearance of type 2 transmigrated canine with GT tract was seen. However, the authors failed to discuss about the GT associated with canine in their report. This could be mainly because most dentists are interested in only anomalies related to tooth or alveolar process and not in normal physiological structures such as GT. In another CBCT report of GT tract by Ahmed et al, ${ }^{17}$ authors have highlighted on the CBCT imaging characteristics of the GT associated with impacted mandibular canine but failed to report the transmigration associated with the canine. Through this, the authors wished to bring light to the fact, that the rare mention of GT in imaging textbooks and articles is merely due to underreporting of the normal incidental findings and not because of the rarity of the condition itself.

Even though GT is a normal structure but it can serve as a source for various pathologic processes arising from the epithelial cell rests of dental lamina within the cord. There are multiple reports of development of adenomatoid odontogenic tumours ${ }^{18}$ from the GT which makes it even more important to report these structures so as to include them in the treatment planning for the impacted tooth and to keep a follow up.

\section{CONCLUSIONS}

GT is a physiologic structure easily appreciated with the advanced imaging modalities. Alterations in eruption of teeth alters the detection and imaging characteristics of GT. Hence, considerable attention should be given to this normal structure on CBCT to understand the physiology of normal and abnormal eruption processes.

\section{REFERENCES}

[1] Marks SC Jr, Schroeder HE. Tooth eruption: theories and facts. Anat Rec 1996;245(2):374-93.

[2] Wise GE, King GJ. Mechanisms of tooth eruption and orthodontic tooth movement. J Dent Res 2008;87(5):41434.

[3] Wang XP. Tooth Eruption without Roots. J Dent Res 2013;92(3):212-4

[4] Ferreira DCA, Fumes AC, Consolaro A, et al. Gubernacular cord and canal - does these anatomical structures play a role in dental eruption? RSBO 2013;10(2):167-71.

[5] Hunter J. The natural history of human teeth. $2^{\text {nd }}$ edn. London: J Johnson 1778.

[6] Malassez ML. Sur la structure du gubernaculum dentis et la theorie paradentaire. Compte Soc Biol 1887.

[7] Nishida I, Oda M, Tanaka T, et al. Detection and imaging characteristics of the gubernaculums tract in children on cone beam and multi-detector CT. Oral Surg Oral Med Oral Pathol Oral Radiol 2015;120(2):E109-E17.

[8] Ando S, Aizawa K, Nakashima T, et al. Transmigration process of the impacted mandibular cuspid. J Nihon Univ Sch Dent 1964;6:66-71.

[9] Almufleh L, Ghori S, Vyas R, et al. Gubernaculum dentis in a transmigrating canine: case report and literature review. Oral Surg Oral Med Oral Pathol Oral Radiol 2019;127(1):e45.

[10] Hodson JJ. The gubernaculum dentis. Dent Pract Dent Rec 1971;21(12):423-8.

[11] Scott JH. The development and function of the dental follicle. Br Dent J 1948;85(9):193-9.

[12] Oda M, Nishida I, Miyamoto I, et al. Characteristics of the gubernaculum tracts in mesiodens and maxillary anterior teeth with delayed eruption on MDCT and CBCT. Oral Surg Oral Med Oral Pathol Oral Radiol 2016;122(4):511-6.

[13] Oda M, Miyamoto I, Nishida I, et al. A spatial association between odontomas and the gubernaculum tracts. Oral Surg Oral Med Oral Pathol Oral Radiol 2016;121(1):91-5. 
[14] Koc N, Dogru HB, Cagirankaya LB, et al. CBCT assessment of gubernacular canals in relation to eruption disturbance and pathologic condition associated with impacted/unerupted teeth. Oral Surg Oral Med Oral Pathol Oral Radiol 2019;127(2):175-84.

[15] Mupparapu M. Patterns of intra-osseous transmigration and ectopic eruption of mandibular canines: review of literature and report of nine additional cases. Dentomaxillofac Radiol 2002; 31(6):355-60.

[16] Gruszka K, Różyło TK, Różyło-Kalinowska I, et al. Transmigration of mandibular canine - case report. Polish Journal of Radiology 2014;79:20-3.
[17] Ahmed J, Tallada AK, Nair A, Shenoy N. Gubernacular cord: an incidental finding on the CBCT scan. International Journal of Advanced Research 2015;3(4):382-5.

[18] Ide F, Mishima K, Kikuchi K, et al. Development and growth of adenomatoid odontogenic tumor related to formation and eruption of teeth. Head and Neck Pathol 2011;5(2):123-32. 